RAE-IC, Revista de la Asociación Española de Investigación de la Comunicación vol. 8, núm. 16 (2021), 195-223 ISSN 2341-2690

Recibido el 29 de junio de 2021 DOI: https://doi.org/10.24137/raeic.8.16.10 Aceptado el 8 de septiembre de 2021

\title{
Herramientas de marketing digital para reorientar la actividad de las oficinas de turismo de Galicia a raíz del COVID-19
}

Digital marketing tools to redirect the activity of Galician tourist offices as a result of COVID-19

\author{
Juncal Suárez, Iria \\ Universidade da Coruña (UDC) \\ iriajuncalsuarez@gmail.com \\ Sánchez-Amboage, Eva \\ Universidade da Coruña (UDC) \\ eva.sanchez.amboage@udc.es
}

Forma de citar este artículo:

Juncal Suárez, I. y Sánchez-Amboage, E. (2021). Herramientas de marketing digital para reorientar la actividad de las oficinas de turismo de Galicia a raíz del COVID-19. RAE-IC, Revista de la Asociación Española de Investigación de la Comunicación, 8(16), 195-223.

https://doi.org/10.24137/raeic.8.16.10

\section{Resumen:}

La irrupción del marketing digital en el sector turístico ha sido esencial en la evolución y mejora de su actividad simplificando muchos de los procesos propios de la mercadotecnia tradicional. Desde la declaración del Estado de Alarma en España a 
mediados de marzo del año 2020 y la consecuente imposibilidad de viajar, parte de los agentes turísticos han comprendido y valorado la utilidad de estas nuevas herramientas para promocionar y comercializar sus productos y servicios turísticos.

El propósito de este trabajo es conocer y proponer las aplicaciones del marketing digital en la etapa de restablecimiento del turismo en Galicia, que puedan aplicarse a las oficinas de turismo de la comunidad gallega. Para ello, se ha recabado información mediante dos técnicas de investigación cualitativa, la observación participante y las entrevistas con expertos, al mismo tiempo que se ha realizado una amplia revisión de la literatura.

La recuperación del sector residirá en la cooperación público-privada para la planificación de estrategias que permitan una supervivencia sostenible del turismo en Galicia. De manera específica, las oficinas de turismo tendrán que aplicarse en la mejora de sus servicios. Para ello, el marketing y la comunicación digital tendrán un papel importante en la recuperación de la demanda con el uso consciente de herramientas como las redes sociales.

Palabras clave: Turismo de Galicia, marketing digital, COVID-19, gestión de crisis, Xacobeo, oficina de información turística.

\section{Abstract:}

The emergence of digital marketing in tourism sector has been essential in the evolution and improvement of everything that comprise its activity. From the State of Alarm that was declared in Spain in mid-March 2020 and the consequent impossibility of traveling, many tourist agents have decided to apply these new tools to promote and market their tourist products and services.

The aim of this research is to know and propose the applications of digital marketing in in a context of change for the Galician tourism sector, which can be applied to the tourism offices of the Galician community. For this, information has been collected through two qualitative research techniques, participant observation and interviews with experts, at the same time as an extensive review of the literature. 
The recovery of the sector will be based on public-private cooperation for the planning of strategies that will allow the sustainable survival of tourism in Galicia. Specifically, the tourist offices of the Camino de Santiago will have to apply themselves to the improvement of their services. For this purpose, marketing and digital communication will play an important role in recovering demand through the conscious use of tools such as social networks.

Keywords: Galician tourism, digital marketing, COVID-19, crisis management, Xacobeo, tourism office information.

\section{INTRODUCCIÓN}

A lo largo de la historia de la humanidad las pandemias han sido una de las mayores preocupaciones de los turistas a la hora de viajar $y$, a diferencia de otras crisis, el coronavirus ha traído consigo la paralización del sector turístico a nivel mundial.

En medio de una fase de contención del virus, con sucesivas olas que obligan a gran parte de las instituciones públicas a endurecer las medidas restrictivas de movilidad y de contacto, las empresas turísticas tienen el foco puesto en la supervivencia de sus negocios y los destinos en conservar el contacto con su demanda.

Por ello, se hace necesario emprender nuevos cambios en la actividad del sector. Algunos de ellos ya se han visto a lo largo de estos meses, la digitalización ha llegado para quedarse definitivamente en el turismo y en eso el marketing digital tiene un papel muy importante, sobre todo, en las nuevas formas de vender y/o promocionar productos y servicios turísticos.

El objetivo general de este trabajo es conocer y proponer las aplicaciones del marketing digital en la etapa de restablecimiento del turismo en Galicia. De forma más específica, buscamos analizar de la mano de diversos expertos en la materia cómo puede ayudar el marketing digital de cara a la gestión de crisis en turismo y qué herramientas pueden mejorar la experiencia del viajero y, más en concreto, la del peregrino en las Oficinas de 
Turismo de Galicia de cara al proceso de recuperación del sector, que coincidirá probablemente con el Xacobeo 2021-22.

A través de la revisión de la literatura existente se crea el marco teórico de la presente investigación, el cual se divide en dos bloques diferenciados, pero que guardan relación entre ellos: por una parte, turismo y marketing digital y, por otro, la gestión de crisis en el sector turístico.

La metodología que se aplicará se fundamenta en dos técnicas de investigación cualitativa. En primer lugar, un ejercicio de observación participante con la finalidad de conocer el uso de las nuevas herramientas comunicativas en la actividad diaria de las oficinas de turismo. En segundo lugar, entrevistas con expertos, a través de las cuales se pretende conseguir una visión más completa de las necesidades actuales en materia de marketing digital en el sector turístico gallego.

Por último, dedicaremos la parte final de la investigación a la aplicación de las nuevas herramientas digitales en las Oficinas de Turismo, las cuales son fundamentales en la cadena informativa y también en la imagen del producto turístico Camino de Santiago y el destino Galicia.

\section{MARCO TEÓRICO}

\subsection{TURISMO Y MARKETING DIGITAL}

La irrupción del marketing digital en el sector ha revolucionado la forma de vender tanto sus productos como sus servicios. A finales de la primera década del siglo XXI, la incursión de internet y la web 2.0 en el turismo supusieron un gran cambio en la manera de viajar gracias al incremento de la competitividad y a la alta diversidad de productos y servicios que aparecen con el acceso a la información y a la "desaparición" de los intermediarios. Además de estas nuevas tendencias, aparece la necesidad de cambio en la manera que compartimos nuestros viajes. Así mismo, nacen las redes sociales que acaban influyendo en muchos de los cambios que hemos citado anteriormente (Domínguez y Araújo, 2014). 
Con la irrupción de la Web 2.0 se produce una apertura importante de las empresas al exterior, lo que repercute en la manera en que estas se relacionan con sus clientes. Según Latorre (2018) definimos el 2.0 como "aquella tecnología web que se basa en comunidades de usuarios y una gama especial de servicios que fomentan la colaboración y el intercambio ágil de información entre los usuarios" (Latorre, 2018, p. 3). Esto ha afectado, en gran medida, a cada uno de los pasos del modelo tradicional de toma de decisiones en la empresa. En sus principios, la creación de diversos blogs y/o páginas corporativas en los que se establecían espacios de comunicación, que posibilitaban el diálogo directo con los clientes y de los cuales la empresa se nutría de información directa y en tiempo real de gran importancia de cara a la toma de decisiones (Dans, 2007).

También resulta disruptiva la nueva promoción turística. Según Domínguez y Araújo (2014), “El producto o servicio turístico ya no se entiende como se venía haciendo hasta ahora, sino que es un concepto que está evolucionando, lo cual obliga de un modo simultáneo a un cambio y adaptación por parte de empresas u organizaciones turísticas, siendo el fenómeno 2.0 y las redes sociales una posibilidad de introducirse y beneficiarse de dicha transición. A las organizaciones turísticas se les abre una gran oportunidad de crear marca, saber lo que piensan los usuarios, incrementar las ventas gracias a la información, independientemente del tamaño de la empresa, sea una pequeña, mediana o gran empresa líder a nivel nacional" (Domínguez y Araújo, 2014, p. 61).

El comportamiento del consumidor ha cambiado y han adquirido mayor importancia aquellas decisiones de compra influidas por las características personales o psicográficas, principalmente por la edad y el momento vital del comprador, ocupación, circunstancias económicas, estilo de vida, personalidad y autoconcepto (Kotler, 2011). Nos encontraremos un turista diferente: informado, exigente, experiencial y con grandes expectativas del destino. Además, que también participa en el proceso de compra directo y en la propia organización del viaje (Altamirano, Túñez y Marín, 2018).

\subsection{VULNERABILIDAD Y RESILIENCIA DEL TURISMO}


El turismo es vulnerable por ser susceptible a sufrir sucesos adversos. Guerras, crisis económicas o ataques terroristas, entre otras, han incrementado la importancia de la seguridad en los viajes. Pero la exposición y sensibilidad del sector a dichos acontecimientos pronto se convirtió, de manera contrastada, en una facilidad de recuperación tal y como pudimos observar en la crisis económica de 2008, la cual afectó principalmente a Estados Unidos y los países de la Eurozona, entre ellos España. A diferencia de otras épocas de depresión, el sector se sobrepuso de manera extraordinaria en un periodo de tiempo corto (Tsao y Ni, 2015 y Quintero, 2016). Tal y cómo apuntó Berengueras en 2016, se atribuyó la celeridad de la recuperación del turismo a los disturbios provocados en Egipto, Túnez y Turquía por la Primavera Árabe, lo que hizo desviar los flujos de demanda hacia nuestro país.

Según Hall, Scott y Gössling (2020) "Las pandemias son claramente una cuestión importante para la sociedad y el turismo, incluso si muchas de sus amenazas potenciales y acciones necesarias para manejarlas han sido ignoradas u olvidadas por el público, los gobiernos y, en ocasiones, por la propia industria" (Hall, Scott y Gössling, 2020, p. 590).

En el caso de España, antes del coronavirus, el riesgo de sufrir una epidemia era prácticamente nulo, aunque con fenómenos como la globalización, podríamos prever que esa amenaza aumentaba y aceleraba su paso hacia nuestro país, sobre todo, cuando aparecían situaciones de caos en otros países como China o Italia.

Nuestro entorno se transformó en cuestión de días, los cambios sucedieron con demasiada rapidez, la pandemia apareció en España en enero de 2020, pero no actuamos hasta marzo de 2020, por lo que el margen de reacción fue muy pequeño cómo para tomar decisiones sin estar bajo presión y con tiempo suficiente para adoptar medidas eficaces (Vargas-Sánchez, 2020).

Con la aparición del primer brote de la pandemia en Asia a finales del año 2019 y la rápida expansión que, a partir de marzo, empezó a afectar de manera mundial a toda la población, aparecieron una serie de nuevas medidas, como los confinamientos o los cierres de fronteras, que han afectado enormemente a la percepción del riesgo, lo cual ha modificado de manera negativa a los viajes del año 2020 (Neuburger y Egger, 2020). 
Sabemos que la percepción de riesgo llega a tener un papel fundamental en la actividad turística porque incide directamente en la demanda. La ausencia de control sobre el riesgo crea intranquilidad en el viajero y le hace detener un viaje si ve que no es seguro, lo que en la presente situación se ha traducido en una fuerte crisis económica en el sector (Vargas-Sánchez, 2020).

Entender, en buena medida, los factores que han llevado al miedo irracional a viajar durante la época COVID, es importante para comprender cómo será el futuro comportamiento del turista para crear acciones de marketing por parte de los destinos y, principalmente, crear información veraz y concisa sobre las políticas de salud pública en los viajes.

Con la aparición de fenómenos como la infoxicación o las innumerables fake news que han infundido el pánico social y el pesimismo, traen consecuencias negativas en cuanto a la intención de viaje. Se ha incidido demasiado en estados de emergencia y no tanto en estados de prevención que podrían haber reducido esa percepción del riesgo. También comentar la observación de comportamientos negacionistas a la situación, que han llegado a poner en peligro la salud del resto de ciudadanos y no han ayudado a la hora de contener el virus (Neuburger y Egger, 2020).

Estos problemas han sido resultado del impacto de la comunicación de crisis y, en parte, de los medios sociales en la percepción de riesgo de la demanda. A pesar de que las continuas crisis han acrecentado el papel de las redes sociales en nuestra vida y que su impacto ha sido indispensable en nuestras tomas de decisiones, pocas empresas han comprendido la utilidad de estos en la gestión de crisis como una vía de dar soluciones rápidas de estabilización, prevención y reconstrucción de un destino (Sigala, 2020).

A nivel empresarial no hay exención en cuanto a la percepción de riesgo, ya que muchas veces este tipo de amenazas cíclicas afecta negativamente al funcionamiento, actividad y reputación de las sociedades turísticas. La disminución contrastada de la demanda trae consigo una inminente interrupción de las llegadas y del gasto turístico. El recelo a dar pasos en falso ante la dudosa evolución del coronavirus, el comportamiento del turista post-Covid y el desconocimiento de las estrategias que llevarán a cabo los gobiernos, 
abre la posibilidad a diferentes situaciones en las que se pueden encontrar el sector privado del turismo a partir de la vacunación masiva de la población (Vargas-Sánchez, 2020a).

Si hablamos de los destinos, las Administraciones Públicas entran en un proceso de reacción ante los perjuicios importantes que la pandemia (en este caso) ha dejado, tanto a nivel sanitario como a nivel económico. Su dimensión se está empezando a reflejar en muchos estudios posteriores a épocas de gran actividad turística como la estival. Como todas las caídas, esta debe tener un sentido constructivo de cara a la prevención de futuros desequilibrios que pueden surgir en el mundo del turismo. Por eso, la gestión de crisis juega un papel fundamental en la reconversión de los destinos y debe convertirse, para todos los agentes que forman parte de la actividad turística, en un proceso de aprendizaje que les permita planificar mejor y crear diferentes instrumentos que faciliten la elaboración de planes de contingencia adaptados a cada situación. Documentos organizativos que prevengan a los territorios de sufrir un alto impacto estructural, personal, presupuestal y demás, para sus administraciones, empresas, etc. (Vargas-Sánchez, 2020).

La globalización ha convertido una crisis que podría haber afectado de manera puntual a un país en otra que ha parado completamente el sector turístico mundial. Por ello, ciertas Administraciones Públicas autonómicas no dudaron en aprovechar para intentar disminuir el impacto negativo de la pandemia sobre el turismo. Con campañas claves perdidas como la Semana Santa, comunidades autónomas como Galicia se pusieron a trabajar en las estrategias a seguir de cara al corto, medio y largo plazo. Por ello, el Plan de Reactivación del sector cultural y turístico, puesto en marcha por la Xunta de Galicia, estableció una serie de medidas para garantizar la adaptación empresarial y la seguridad del turismo receptor (Araújo, Fraiz, y Toubes, 2020).

Posteriormente, en la antesala del Año Xacobeo 2021-2022, nació también la necesidad de planificar el período de recuperación del sector. Por ello, gracias a la cooperación público-privada se ha elaborado el Plan director 2021-2023 Galicia Destino Seguro. 
Este documento redefinirá el rumbo del turismo en Galicia con el que se busca afrontar los retos que ha dejado la pandemia del coronavirus y reactivar el sector y el empleo turístico. A la par también sigue en ejecución el Plan Estratégico de Galicia 2015-2020 con el que la Administración seguirá fomentando el modelo turístico sostenible, de calidad y selectivo (Xunta de Galicia, 2020)

Se hace indispensable la percepción de confianza y calidad por parte del viajero, además de aportar una mayor especialización y modernización digital. Por otro lado, también será importante, a nivel promocional y comercial de los destinos y productos turísticos, los sellos o certificados de salud y seguridad (Turismo de Galicia, 2020 y Xunta de Galicia, 2021).

Por lo tanto, tal y como apunta Vargas-Sánchez (2020), “La gestión de crisis es, por tanto, un problema estratégico que todos los destinos turísticos deben ocuparse de gestionar adecuadamente y no deberían subestimar. Si no estaba en sus agendas, es el momento de abrirle paso en ellas, sin más dilación" (Vargas-Sánchez, 2020, p. 17). Por todo ello, la recuperación económica de nuestro sector y del resto tendrá que traer consigo una serie de cambios estructurales que supongan, según Quintero (2016), "un aumento significativo de la inversión en investigación, desarrollo e innovación ( $1+D+i)$, así como, innovación en energías verdes o renovables, sanidad, transporte y pequeñas y medianas industrias tecnológicas" (Quintero, 2016, p. 29).

Esta crisis debe ser el punto de inflexión definitivo para promover el desarrollo sostenible de los países, pero también de su actividad turística mediante la gestión de crisis y su planificación estratégica a nivel promocional y de comercialización (Polo, 2020).

\subsection{LA UTILIDAD DEL MARKETING DIGITAL EN LA GESTIÓN DE CRISIS TURÍSTICA}

Se hace conveniente el comprender la divergencia entre periodos de depresión anteriores para establecer mejores soluciones de marketing y promoción. Al cobrar importancia la relación turista-destino, como oferentes de experiencias turísticas, debemos analizar las fluctuaciones del posicionamiento de nuestra marca durante esa etapa que forma parte de un estudio previo a nivel interno y posteriormente elaborar 
los "planes de acción a corto, medio y largo plazo" con el apoyo de todos los agentes del sector.

El período de confinamiento resultó para muchos un momento de reflexión en el que valorar de manera más contrastada el trato humano dentro de la era digital en la que nos encontramos. Aunque sabemos que muchos destinos han tenido que adaptar su propuesta de valor a lo digital por la restricción de movilidad, ha sido la única alternativa posible para mantenerse en contacto directo con su demanda turística (Figueroa y Gómez-Zorrilla, 2020).

Durante estos cierres, la gente ha consumido experiencias turísticas virtuales, lo que ha supuesto una mayor utilización y familiarización con las nuevas tecnologías y la posibilidad de adoptarlas como algo más habitual en su vida diaria. En definitiva, la mentalidad de muchos de nuestros antiguos viajeros ya no será igual (Sigala, 2020).

La gran dependencia que la demanda turística ha tenido con las redes sociales durante el Estado de Alarma ha venido también "justificada" por su uso intensivo en las empresas y en los destinos para retener su demanda durante su período de inactividad. En gran parte de los casos, la oferta se ha dado cuenta del potencial de este tipo de herramientas digitales de cara a la venta y promoción de sus productos y servicios.

Por una parte, los efectos han sido positivos ya que son un canal inmediato y efectivo para proporcionar y recibir información instantánea en casos de emergencia, lo que ha facilitado en muchos casos tomas de decisiones de reacción más rápidas y efectivas.

Por otro lado, han surgido problemáticas de falta de criterio y de excesiva divulgación de información durante un corto tramo de tiempo, lo que ha proliferado fenómenos como la infoxicación o las fake news (Calleja-Reina, Rojano y Mas, 2018).

En definitiva, los medios sociales han cambiado la forma en que nos comunicamos, sobre todo, en situaciones de mayor tráfico de datos y creación de contenido para las tomas de decisiones. Estos momentos han servido para que todos los agentes del sector turístico trabajasen de manera colectiva, se anticipasen y, en ciertos casos, mitigasen efectos adversos de las crisis (Antony y Jacob, 2019). 
No cabe duda de que el marketing digital ha mejorado muchos aspectos el sector turístico, uno de estos ha sido la alta competitividad que ha adquirido desde la irrupción de las nuevas tecnologías. Antes de la pandemia, las Administraciones se dedicaban a invertir grandes cantidades de dinero en estrategias de desarrollo de los destinos y en la promoción de sus productos y servicios turísticos.

Al contar con una gran financiación y confianza por parte de diferentes agentes del sector, se hacía esencial la creación de una estrategia de marketing digital que se adecuase al mensaje de la Administración y al público objetivo al que se querían dirigir creando contenido creativo, viral y de buena calidad (Prokopenko, 2019)

Con la incursión del coronavirus y la consecuente paralización del turismo, tanto destinos como empresas deberán tener la capacidad de adoptar herramientas digitales en sus procesos de recuperación para conocer de primera mano las necesidades de sus viajeros y hacer un seguimiento de estas.

La adopción de herramientas digitales de gestión en el sector, promueven la creación de nuevas profesiones, productos y servicios turísticos más sostenibles que promueven el contacto del viajero con la comunidad local, las industrias creativas propias y los viajes seguros mediante el uso de la tecnología. Por ello, herramientas de marketing digital como la Inteligencia Artificial y el Big Data, serán claves a la hora de gestionar el turismo de masas, proteger a la población local y los recursos turísticos de los destinos (ONU, 2020).

Cada vez se diversifican más las técnicas utilizadas en la promoción turística desde la creación de la página web, todo ello ha ido evolucionando con el SEO y SEM, el marketing de contenidos, el email marketing, etc. hasta llegar a otras tendencias como los asistentes de voz, el whatsapp business, el videomarketing o, el que ha marcado un antes y un después en la forma de comunicar y atraer turismo, el marketing de influencia y redes sociales (Prokopenko, 2019).

Para promocionar un destino, debemos tener claro que será necesario conocer los segmentos a los que nos dirigimos, analizar el entorno interno y externo del destino y 
establecer una serie de objetivos a alcanzar, porque en ello residirá el éxito de la elección y la utilidad de las herramientas de marketing digital en la promoción de la actividad turística.

\section{METOdOLOGÍA}

Para el caso que nos ocupa, el apartado de metodología se estructura en base a las dos técnicas que permitirán alcanzar el objetivo propuesto: observación participante y entrevista personal semiestructurada con expertos.

\subsection{OBSERVACIÓN PARTICIPANTE}

En la realización de esta técnica cualitativa, se colaboró de manera activa, entre los meses de julio y noviembre del año 2020, en la Oficina de Información del Camino de Santiago, ubicada en Calle Carretas 33 en la ciudad de Santiago de Compostela, gracias a la bolsa de formación ofrecida por la Agencia de Turismo de Galicia.

Se evalúa el entorno del punto de información turística seleccionado y se identifica una serie de errores que, en base a la literatura y a la experiencia previa requieren de atención y actuación.

\subsection{ENTREVISTA PERSONAL SEMIESTRUCTURADA}

Para la realización de las entrevistas, se ha contado con la colaboración de expertos en materia de turismo, marketing digital y gestión de crisis. En total, se ha contactado vía email con ocho especialistas de los ámbitos mencionados: cuatro nacionales y cuatro gallegos. La finalidad de esta selección ha sido obtener una visión global y diversificada del sector.

- Investigación:

- Entrevistado 1: Coordinador del Máster de Dirección y Planificación del Turismo de la Universidade de Vigo.

- Entrevistado 2: Doctor en Gestión y Dirección de Empresas de la Universidade de Vigo y experto en la gestión de crisis en el sector turístico. 
- Empresas:

○ Entrevistado 3: Comunicador en Hosteltur.com

○ Entrevistado 4: CEO de Latexos de Turismo

○ Entrevistado 5: CEO de Pilgrim

- Entrevistado 6: CEO de Hosperience

- Entrevistado 7: Consultora de marketing y turismo digital

- Destinos:

- Entrevistado 8: Gerente de Visit Benidorm

El proceso de las entrevistas se ha realizado a lo largo de la semana del 18 al 22 de enero del 2021. Debido a las restricciones del Estado de Alarma, motivadas por la pandemia COVID-19, el método empleado para la obtención de las respuestas ha sido en modo conversación con la herramienta telemática de videoconferencias Skype y vía escrita por Google Forms. En el primer caso, fue necesaria la grabación del proceso para posteriormente realizar la transcripción de las entrevistas.

En cuanto a la forma, la conversación estaba dividida en 13 preguntas y estructurada en 5 bloques. En primer lugar, con información más general (turismo y marketing digital) y posteriormente más específica (gestión de crisis en el turismo y su relación con el marketing digital, la crisis del coronavirus y el Xacobeo 2021-2022).

Primeramente, se han redactado de manera manual todas las conversaciones. Posteriormente, para organizar y extraer la información más relevante, se ha utilizado el programa MAXQDA que está basado en la teoría fundamentada, aquella que se obtiene a través de datos cualitativos aportados por técnicas como las entrevistas semiestructuradas (Cuñat, 2007).

\section{RESULTADOS}

\subsection{RESULTADOS DE LA OBSERVACIÓN PARTICIPANTE Y DE LAS ENTREVISTAS CON EXPERTOS}


Tras la observación participante durante el periodo de inmersión en la Oficina de Información del Camino de Santiago, ubicada en Calle Carretas 33 en la ciudad de Santiago de Compostela, se detectan ciertos aspectos a mejorar que se exponen a continuación.

- Utilización de herramientas digitales no oficiales y, por lo tanto, desconocimiento de las herramientas oficiales.

- Escasa visibilidad de las oficinas de turismo en el entorno web.

- Inexistente intercomunicación con el resto de las oficinas de turismo enmarcadas en el Camino de Santiago.

- No se dispone de folletos en formato digital en la oficina.

- Solo se dispone del formato papel para realizar encuestas al peregrino.

- La página de albergues públicos de Galicia no dispone de información en tiempo real de las habitaciones disponibles en el territorio.

A la observación participante, se une la opinión de los expertos. En este sentido, sobre el papel del marketing digital en el sector turístico, gran parte opinan que es fundamental en la promoción y comercialización del turismo.

Con respecto a los cambios que ha sufrido el turismo desde la irrupción del marketing digital, todos consideran que el sector ha sufrido una transformación indudable en aspectos como el ciclo y comportamiento de compra, la creciente importancia del turista, los viajes más experienciales o la aparición de nuevas profesiones, entre otros.

Si nos referimos a la crisis del coronavirus, la mayor parte sopesa que acontecimientos como el confinamiento domiciliario han favorecido la democratización y aceleración del proceso de digitalización del sector a través del consumo de nuevos productos turísticos, como los viajes o visitas virtuales. El uso del marketing digital durante la pandemia tendrá consecuencias en el futuro del sector porque han cambiado nuestras rutinas y la propia tecnología.

En cuanto a la gestión de crisis en turismo, todos tienen claro que este tipo de desequilibrios temporales afectan de manera directa e indirecta al turismo y a otras 
actividades económicas. Entre los efectos relacionados con la temática de esta investigación están: la caída de la inversión empresarial en captación de clientes a través de la publicidad de pago, disminución del gasto turístico o desviación de flujos de viajeros hacia destinos con oferta similar. Sin embargo, también forman parte de un proceso de nuevas oportunidades y aprendizajes de cara al futuro del turismo: analizando las necesidades de los segmentos, repensando las acciones bien y mal hechas o estableciendo una serie de propuestas de mejora, entre otras.

Será necesario desarrollar planes de contingencia o estratégicos de cara a futuras crisis, todos los entrevistados concuerdan en la necesidad de elaborar este tipo de documentos de planificación para adquirir competencias en su gestión y posterior ejecución, poniendo en valor la importancia de la cooperación público-privada en el proceso de gestión y su puesta en práctica. Concretamente, inciden en elaborar los proyectos con criterio, basándose en la posibilidad de que ocurra y su grado de impacto. También, estiman que es necesario contar con profesionales formados y especializados en este tipo de ámbitos que actúen de manera consciente en el proceso de planificación y de comunicación.

Acerca del papel que tiene el marketing digital en la gestión de crisis en turismo, gran parte de los expertos destacan que ha sido esencial la creación de campañas de comunicación recíprocas, personalizadas y flexibles. Parte de ellos han concretado que herramientas como el email marketing o las redes sociales, entre otras, han facilitado que este tipo de promociones sean más cercanas, naturales y humanas. Opinan que el marketing digital ha sido esencial en la comunicación de las empresas y los destinos, ya que ha servido de "altavoz" en un momento en el que, a pesar de la gran crisis que estaba sufriendo el sector, era necesario promocionar y dar a conocer lugares y destinos seguros de cara a épocas mejores.

Sobre si las redes sociales son una herramienta efectiva a la hora de gestionar una crisis, la mayor parte de los expertos contestó que, principalmente, son útiles en momentos en los que se necesita recibir feedback instantáneo para actuar de manera inmediata y transmitir valores mediante la adaptación del lenguaje utilizado en los mensajes, posts, etc. 
En relación con las problemáticas de cara a la recuperación de la demanda turística en el Xacobeo 2021-2022, todos los entrevistados concuerdan que el principal problema es la actual pandemia. Gran parte de ellos inciden en la necesidad de comprender la percepción de riesgo y seguridad sanitaria del viajero después del coronavirus. Esto hace referencia a que la demanda tenga la posibilidad económica (renta disponible) y sentimental (deseo) para volver a viajar después de esta época de incertidumbre e inseguridad.

En lo tocante a las estrategias de marketing que se plantearían de cara a esa recuperación, por una parte, consideran que es necesario crear estrategias de producto, servicio y destino seguro, personalizados y de calidad/precio, tanto en disfrute como en comunicación para generar experiencias con gran implicación emocional de cara al fenómeno Año Santo Xacobeo. Por otro, concuerdan en la parte "sincera y honesta" del marketing, recomendando no viajar durante los períodos de confinamiento.

De la misma forma, si preguntamos sobre las herramientas de marketing digital que se han hecho indispensables con la presente crisis, todos los entrevistados están de acuerdo que las redes sociales son una de las que más se han utilizado. Seguidamente se posicionan el email marketing, el Big Data y la analítica web, aquellos instrumentos que permitan conocer la evolución de la capacidad aérea, la evolución de las reservas, la ocupación de los alojamientos, la opinión sobre productos, el interés por mercados, y zonas geográficas más pequeñas. En definitiva, recopilar y extraer datos relevantes de los viajeros que nos faciliten la toma de decisiones en la planificación a corto, medio y largo plazo. Acerca del modo de aplicación de estas herramientas anteriores, inciden en la importancia de conocer a los colectivos y segmentos a los que nos vamos a dirigir y elaborar las estrategias que se adapten a esa potencial demanda.

En lo relativo a si es necesario repensar el turismo, la mayoría de los entrevistados estuvieron de acuerdo en la necesidad de cambio en la industria. Varios de los entrevistados consideran que es la ocasión de poner en valor recursos propios, crear iniciativas más verdes y buscar la esencia de los destinos. 
Finalmente, con el objetivo de conocer cómo será el sector, se les propuso la pregunta de cómo veían el sector dentro de 5 años a la cual cada uno aportó sus reflexiones personales sobre el futuro del turismo, pero la gran mayoría y de forma optimista opina que la industria de la felicidad estará recuperada.

\subsection{PROPUESTAS DE MEJORA}

Se exponen los aspectos generales a mejorar en el entorno de las oficinas de turismo de Galicia tras la observación participante realizada y la interpretación de la información, que hemos podido recabar de la opinión de los ocho expertos. De esta forma se elaboran propuestas o posibles soluciones para la etapa de recuperación de la actividad turística después del coronavirus.

Siguiendo los resultados de la observación y la entrevistas, se detecta que gran parte de los turistas están familiarizados con las nuevas tecnologías, por ello utilizan herramientas digitales externas. Concretamente los peregrinos que caminan a Santiago de Compostela suelen utilizar las aplicaciones Gronze o Camino de Santiago Eroski, por lo que se hace necesaria una mejora de los servicios de la App del propio camino, para personalizar su ruta, contactar con otros peregrinos, reportar incidencias a las oficinas/albergues del camino y sistema de geolocalización). Esto podría llevarse a cabo con un proyecto en el que prime la cooperación público-privada con empresas que trabajan con este tipo de herramientas actualmente (Figura 1) en el sector turístico gallego.

También, la mayor parte de los caminantes no conocen la aplicación oficial, por lo que se tendría que visibilizar más y así aprovecharla para obtener datos ofrecidos por el peregrino. Por ejemplo, se puede facilitar un QR (Figura 2) con el enlace de descarga a Google Play y App Store.

La escasa visibilidad de las oficinas de turismo en la web dificulta el encontrar las especificaciones de servicios, los números o el email de contacto. Por ello, sería aconsejable la creación de un apartado que muestre directorio de números y correos (Figura 3) que faciliten el contacto directo con las oficinas de información de Galicia. Todo ello, dentro de la landing page. 
Figura 1. Ejemplo de posibles facilidades que podría tener la app del camino

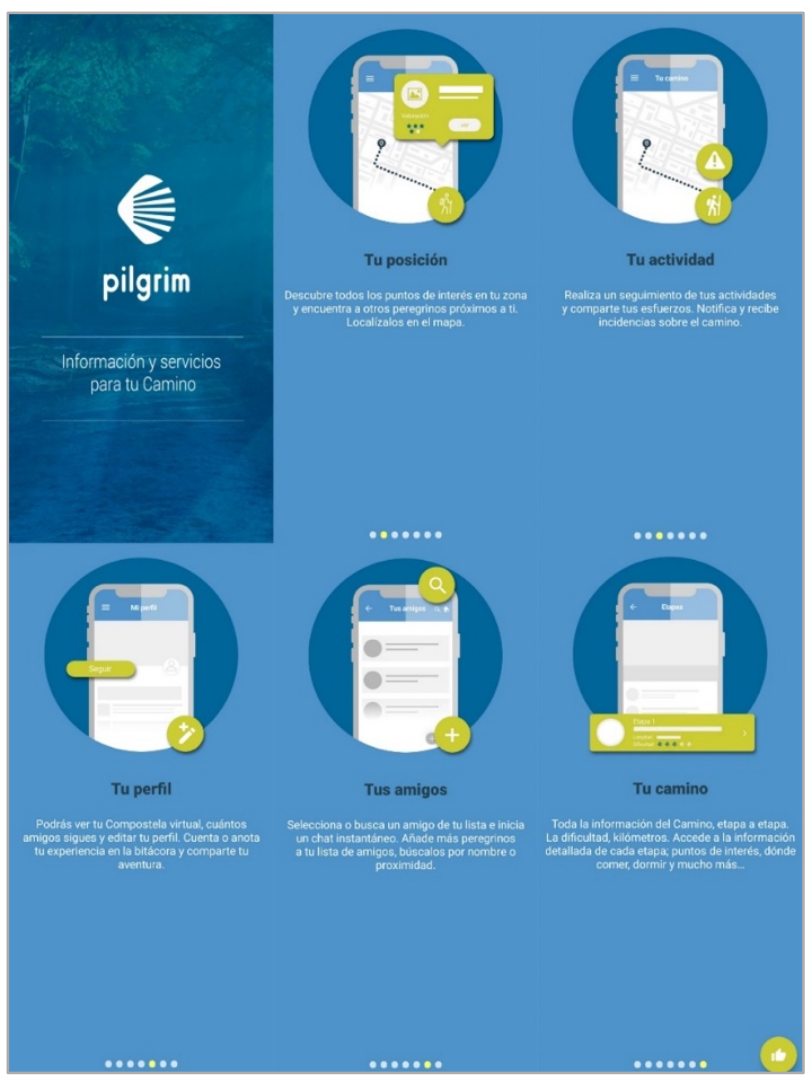

Fuente: Pilgrim App (https://www.pilgrim.es/pilgrim-app/)

Figura 2. Modelo QR descarga directa App Oficial Camino de Santiago

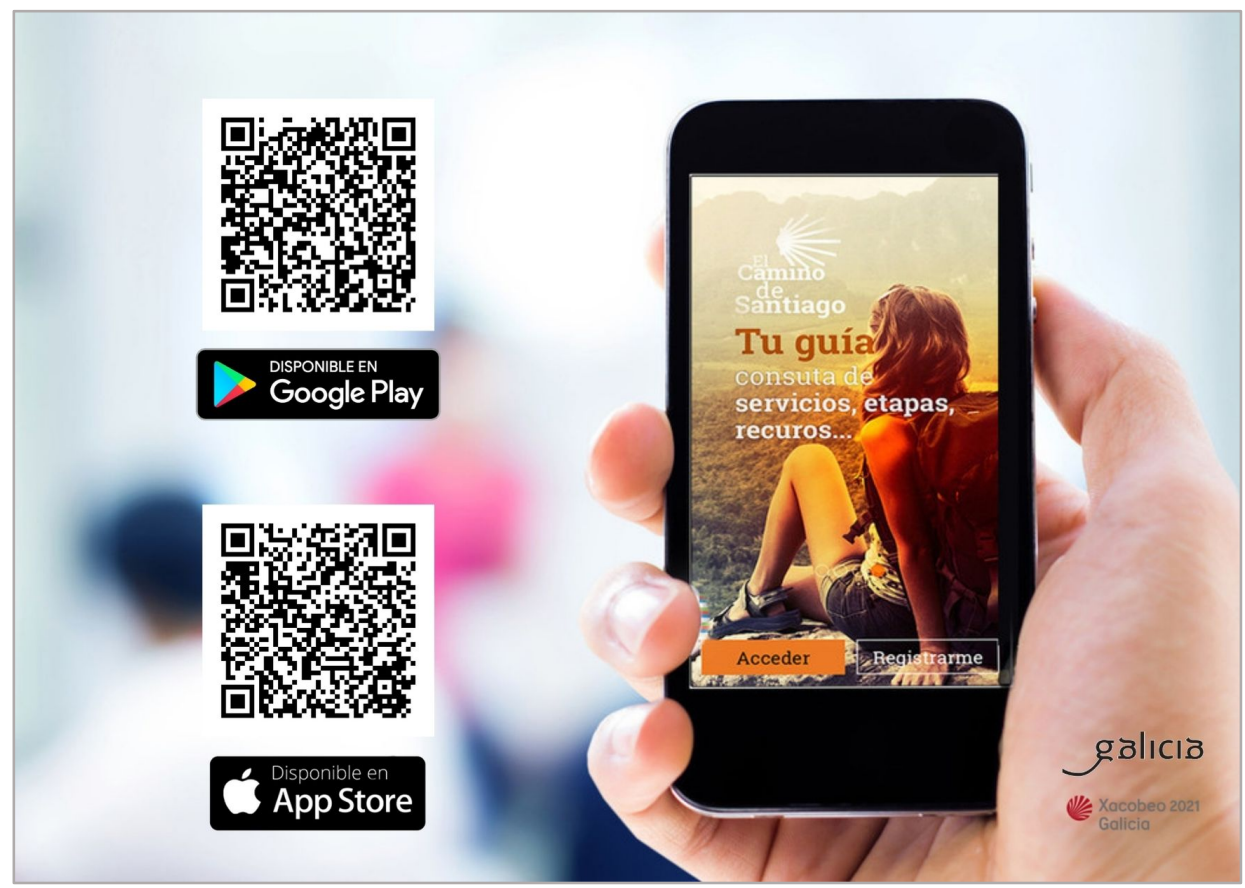

Fuente: https://www.caminodesantiago.gal/es/inicio y editada con Canva

RAE-IC, Revista de la Asociación Española de Investigación de la Comunicación vol. 8, núm. 16 (2021), 195-223 
Figura 3. Landing page de Turismo de Galicia

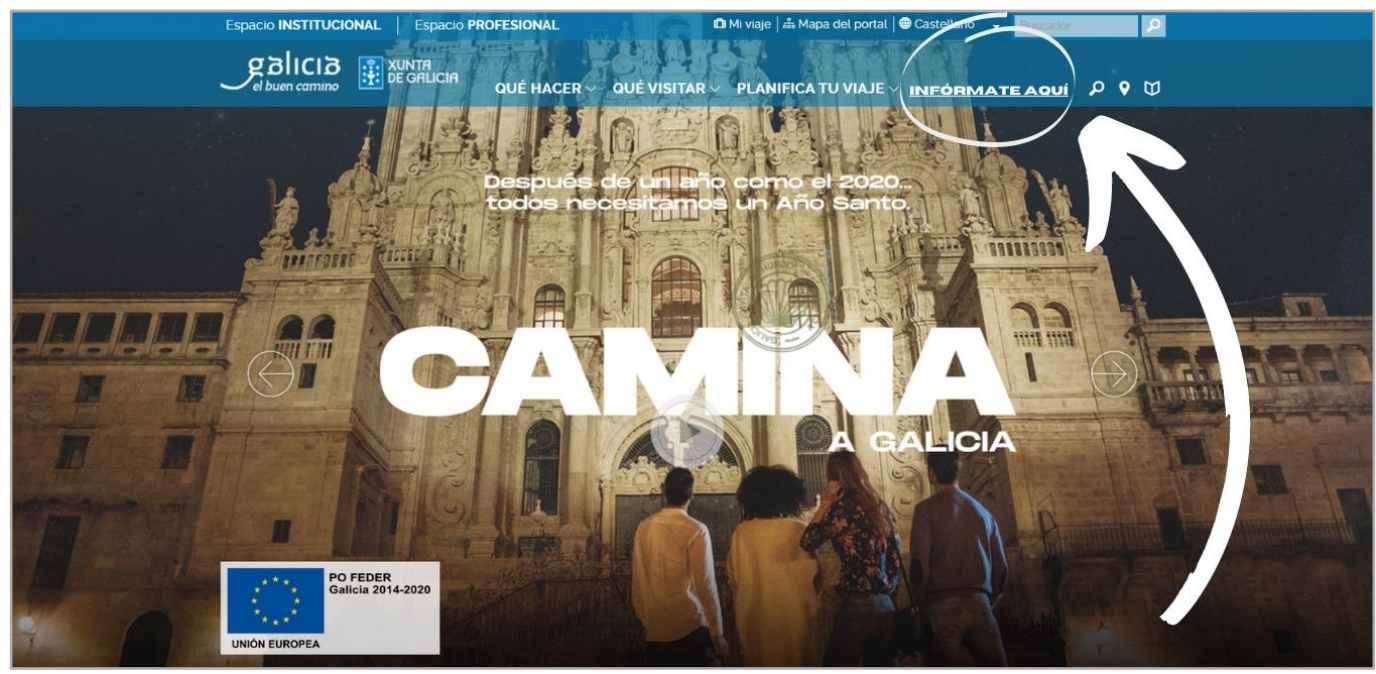

Fuente: Imagen extraída de la Página oficial de Turismo de Galicia (https://www.turismo.gal/inicio) y editada con Canva

Otro ejemplo podría ser la creación de un perfil en la aplicación de mensajería WhatsApp Business (Figura 4) que facilitase el contacto directo y recíproco con el peregrino.

Figura 4. Prototipo perfil de la Oficina de Turismo de Carretas

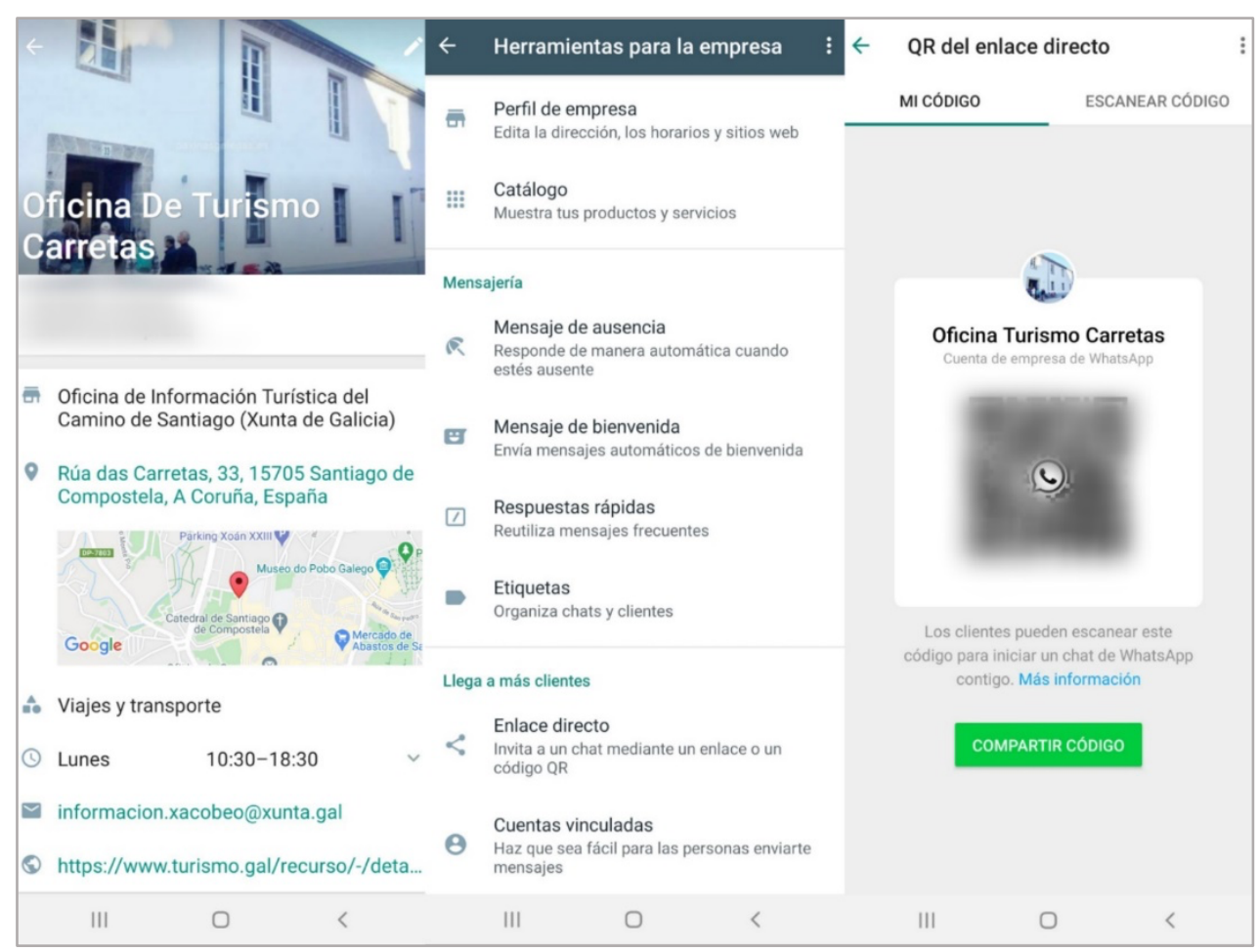

Fuente: Captura de pantalla extraída de la aplicación WhatsApp Business e información extraída de la Página oficial de Turismo de Galicia (https://www.turismo.gal/inicio) 
Se percibe una casi inexistente intercomunicación con el resto de las oficinas de turismo enmarcadas en el Camino de Santiago. Con que, gracias a la tecnología se podría construir una plataforma o intranet donde cada oficina se pueda crear una red de contacto instantáneo entre estas (Figura 5).

Con la crisis del coronavirus se ha hecho esencial el contacto de la red de oficinas por posibles brotes que pudiesen surgir en el camino. En muchas ocasiones, la incertidumbre sobre los posibles casos de COVID-19 ha creado inseguridad entre los peregrinos que realizaban sus respectivas rutas en el mismo periodo de tiempo (Lugilde, 2020).

Figura 5. Prototipo Intranet de las Oficinas del Camino de Santiago

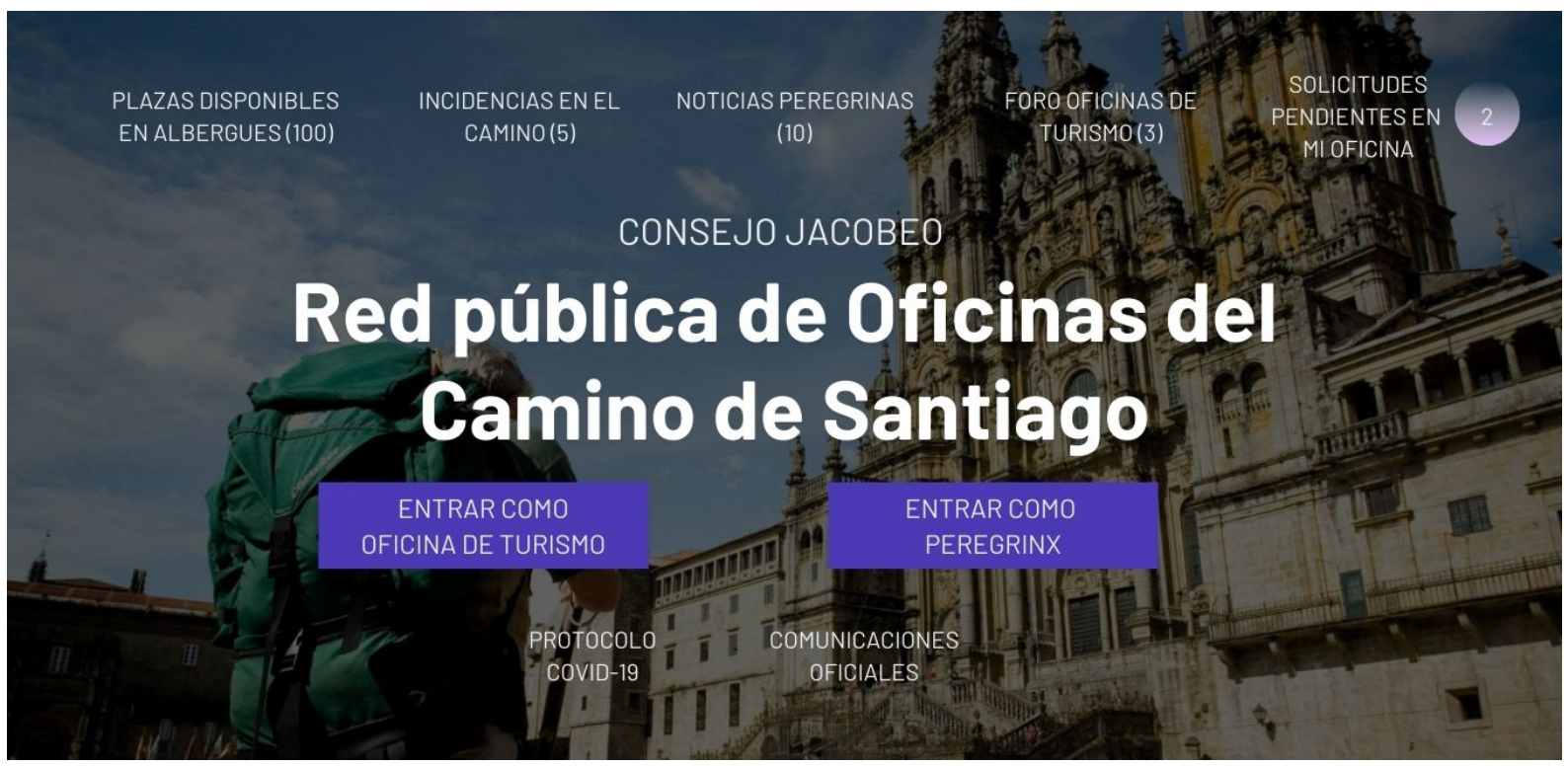

Fuente: Elaboración propia a través de Canva

No se dispone de folletos en formato digital en la oficina de manera que se podría crear códigos QR que estuviesen expuestos en la oficina con el fin de digitalizar documentos (Figura 6). Esto no supondría la eliminación del formato en papel, debido al perfil de peregrino que realiza el camino que se enmarca en edades avanzadas y también, por la gran brecha digital que sigue existiendo hoy en día. 
Figura 6. Modelo QR folleto digital Camino Portugués Interior y de la Costa

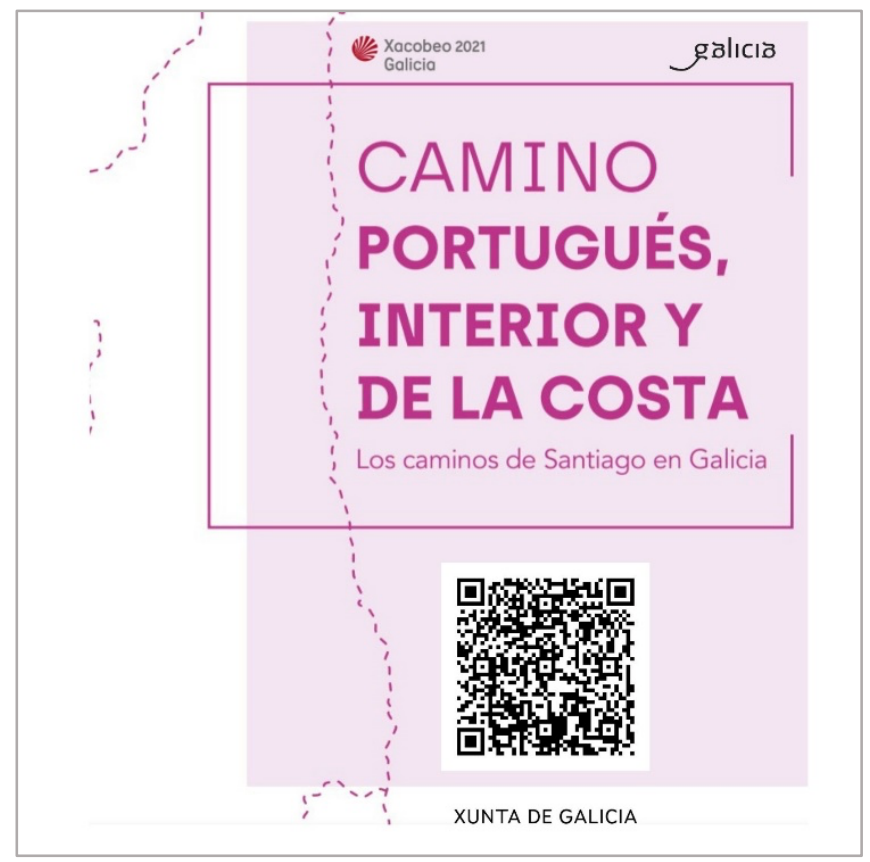

Fuente: Imagen extraída de la página oficial del Camino de Santiago (https://www.caminodesantiago.gal/es/inicio) y editada con Canva

Se recaban los datos de los peregrinos para la realización de las estadísticas en papel, lo que supone un gran gasto innecesario de hojas para luego transcribirlas a ordenador. Para simplificar los procesos, se podría poner a disposición un Código QR con el que puedan descargar flyers, mapas, folletos cumplimentando los datos necesarios para realizar las estadísticas mensuales (camino que realizan, personas que lo realizan, procedencia e información que solicitan).

La página de albergues públicos de Galicia no dispone de información en tiempo real de las habitaciones disponibles. Por lo que sería necesario, posibilitar dentro de la plataforma anteriormente mencionada el contacto directo entre los albergues y las oficinas, ofreciendo información en tiempo real, tanto de la apertura del establecimiento como de las habitaciones que están disponibles (Xunta de Galicia, 2020a).

Se percibe cierta "turismofobia" por parte de la población que rodea el Camino Francés, (Pombo, 2017) aunque está surgiendo en otras rutas que no están tan explotadas, como el Camino de Invierno, ruta que transcurre mayoritariamente por la Ribeira Sacra. Por ello se hace necesario concienciar a la población local que la actividad turística es un 
todo que aporta riqueza al pequeño comercio, así como da a conocer la cultura endémica del vino, entre otros (Cortés, 2020).

En ciertos casos, se han observado publicaciones de información errónea sobre recursos y oferta turística en la página oficial de Turismo de Galicia (Araújo, 2020). Por consiguiente, es fundamental revisar y actualizar los datos expuestos contrastándolos con los propios agentes locales de los destinos (E.A.E., 2020).

Aunque el usuario Turismo de Galicia está activo en redes sociales como Facebook o Instagram en las cuales es necesaria una mayor interacción por parte de esta institución, sigue percibiéndose poca presencia en nuevas redes sociales como TikTok (Sánchez, 2020). Por esto, sería innovador incluir una serie de prescriptores oficiales que guarden un fuerte vínculo con Galicia y su comunidad local, jóvenes y procedentes de diversos ámbitos como: el deporte, la literatura, la música e incluso el propio entorno de las redes (Figura 7). De cara a fenómenos como el Xacobeo los elegidos serían Ana Peleteiro, Michael Chenlo y Fredi Leis, entre otros.

Figura 7. Propuesta de prescriptores de la marca Galicia y del Camino

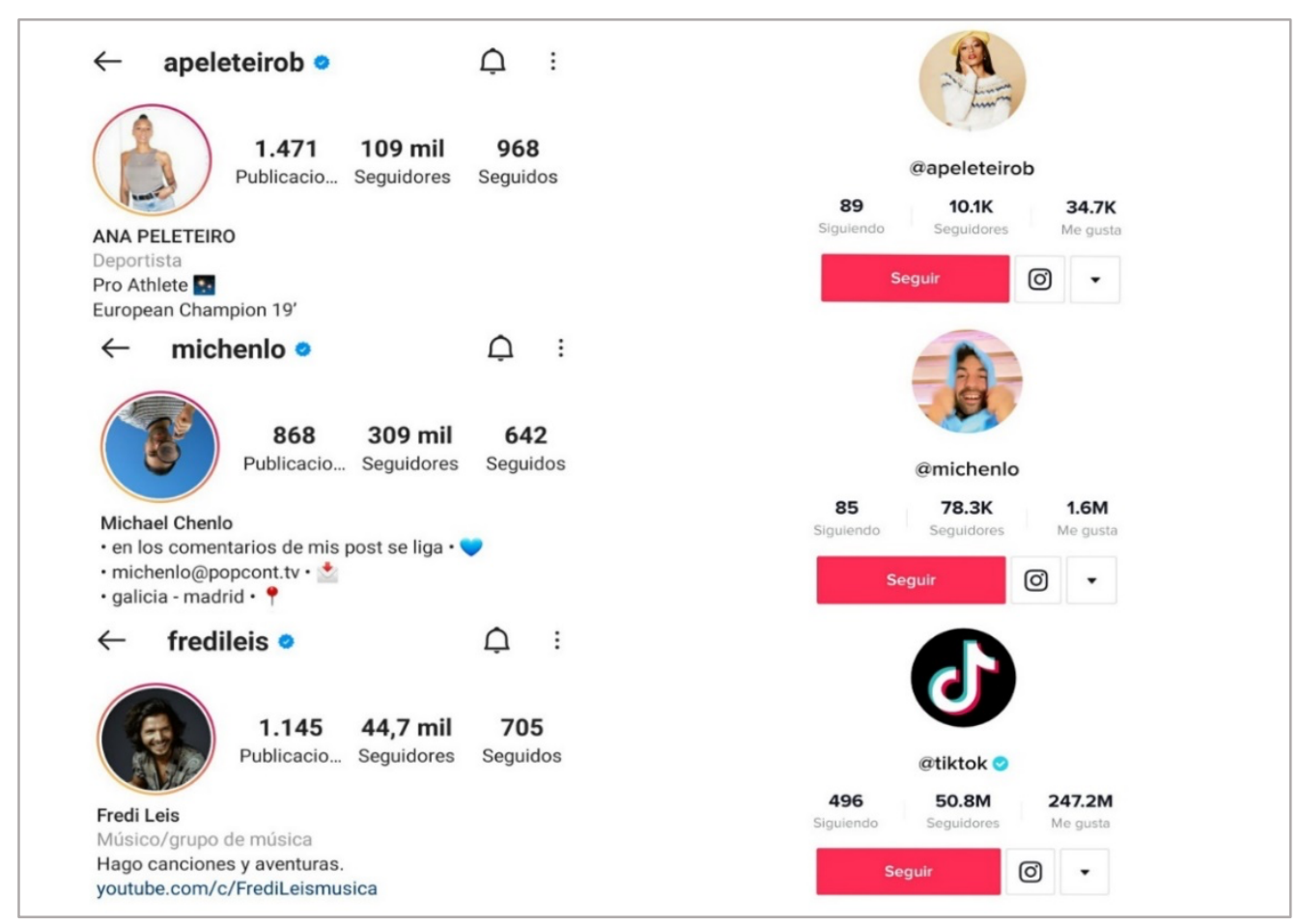

Fuente: Capturas de pantalla extraídas de las herramientas sociales Instagram y Tik Tok 
Se ha observado en los últimos años una gran inversión en producciones audiovisuales que no consiguen reflejar la verdadera esencia de Galicia. Por lo tanto, se tendrá que apostar por otro tipo de medios que la comunidad local valora más positivamente como los reportajes en revistas de viaje como Condé Nast Traveler, donde los artículos relacionados con nuestra tierra los elabora un periodista de la propia Comunidad Autónoma (Amado, 2020) o colaboraciones con travelbloggers con fama internacional y que estén creando contenido de valor para sus seguidores como pueden ser, sin duda, Adrián y Gosia en su blog, canal de YouTube o perfil de Instagram (Molaviajar, 2020).

Como inciso, hay que destacar también una tendencia al alza en el año 2020 y que ha tenido mucho éxito en diversas plataformas como Spotify, el podcast (Figura 8). Un nuevo formato al que muchas marcas y destinos se han apuntado.

Figura 8. El podcast como herramienta tendencia en el marketing digital

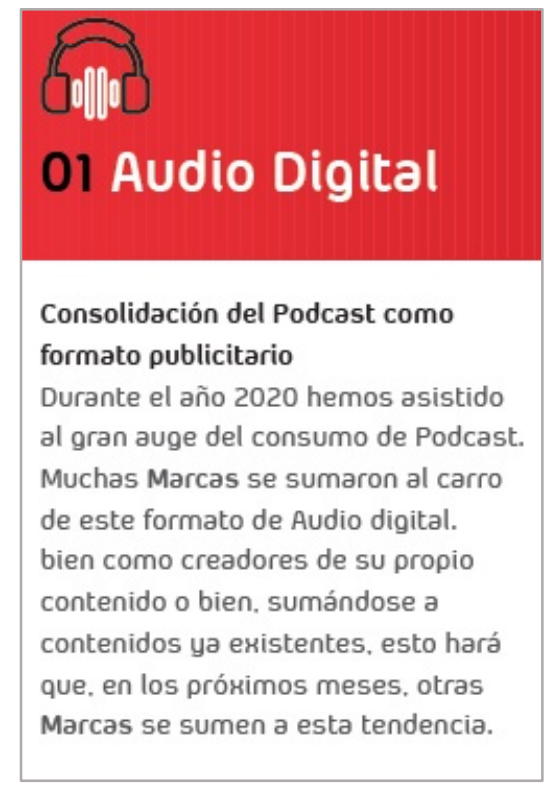

Fuente: Captura de pantalla extraída de IAB Spain (2021)

Todas las propuestas anteriores han sido elaboradas en base a varios factores: la experiencia personal, el proceso de observación participante y las entrevistas de expertos del sector. Con lo que, en base a los resultados obtenidos, podemos corroborar que el uso del marketing digital ha sido esencial desde su propia incursión en el turismo gallego, digitalizando y democratizando gran parte de los procesos: desde la creación hasta el consumo del producto o servicio turístico. 
Si centramos nuestra investigación específicamente en las oficinas de turismo, se hace fundamental la introducción y consecuente aplicación de este tipo de herramientas digitales en la actividad diaria de los servicios de información turística ubicados en las rutas jacobeas.

Esto no significa suprimir las oficinas físicas, sino revalorizar y visibilizar su importante labor dentro del sector, devolviéndole también su relevancia a la hora de realizar una planificación de crisis efectiva. En la cual, hay que destacar su valor comunicativo que forma parte de la imagen del producto Camino de Santiago y de la marca Galicia.

\section{CONCLUSIONES}

Tanto empresas como clientes se han adaptado a la nueva realidad digital de nuestra era. A nivel empresarial, se ha observado un gran esfuerzo de adaptación de las empresas de cara a las nuevas demandas de los clientes. Por otra parte, el consumidor ha ido modificando sus hábitos y entornos en base a la evolución de la tecnología en su experiencia vital, tanto a nivel personal como profesional, algo que seguirá definiendo al consumidor del siglo XXI.

La irrupción de una pandemia mundial no ha hecho más que reafirmar que el uso de la tecnología es uno de los elementos que va a marcar el cambio de ciclo en el modelo turístico, convirtiendo los períodos de crisis en oportunidades de replanteamiento y mejora de la actividad a todos los niveles.

Pero ¿cómo será el turismo después del coronavirus?

Según los expertos, con la situación actual, España podría perder gran parte de su oferta turística, no en recursos sino en empresas, algo que puede llegar a disminuir la demanda. Con lo cual, todos los agentes del sector deberían cuestionarse si su oferta es adaptable a las nuevas exigencias de los viajeros y a las condiciones de la nueva era de convivencia con la pandemia. Será necesario planificar de manera conjunta las estrategias que ayuden en la supervivencia "emocional y racional" de la actividad turística a nivel económico, social y medioambiental, después de un largo tiempo de paralización. Del mismo modo, también destacan que estos cambios tendrán que venir 
con un mayor uso de la tecnología y de nuevas herramientas como el Big Data o la analítica web, que permitan una mejor gestión de reanimación de la industria del turismo. Por ello, la comunicación digital se sitúa en primer plano como recurso necesario a la hora de gestionar la crisis y, en consecuencia, recuperar la demanda turística en fases avanzadas de la pandemia (como la vacunación masiva y la consecuente inmunidad de grupo).

Concretamente, el marketing digital podría tener la capacidad de aportar propuestas de valor a los destinos en cuanto a la desviación de flujos, mediante la adaptación o personalización de la oferta turística a cada viajero o colectivo de viajeros según sus intereses.

Con todo, si enfocamos el radio de actuación en las oficinas de turismo, es imprescindible revalorizar y visibilizar su papel dentro de la cadena de valor del sector. La digitalización de muchos de sus procesos no significa la supresión del punto de información físico, sino renovar su imagen y destacar su valor comunicativo esencial en la imagen del Camino de Santiago y, en general, de Galicia. De manera que los entes competentes podrían sopesar la posibilidad de introducir y aplicar las herramientas digitales recomendadas en apartados anteriores y que, sin duda, devolverán la importancia a las oficinas de turismo como agente participante activo de la planificación y gestión de crisis.

\section{REFERENCIAS BIBLIOGRÁFICAS}

Altamirano Benítez, V. P., Túñez López, J. M. y Marín Gutiérrez, I. (2018). Turista 2.0, comportamiento y uso de los medios sociales. Chasqui. Revista Latinoamericana de Comunicación, 209-225. http://hdl.handle.net/10347/22389

Amado, R. (2020, 24 septiembre). Carta de amor a Galicia. Traveler. https://www.traveler.es/viajeros/articulos/carta-de-amor-a-galicia/19033

Antony, J. K. y Jacob, J. M. (2019). Crisis Management in the Tourism Industry-The Role of Social Media Platforms. Atna - Journal of Tourism Studies, 14(1), 83-96. https://doi.org/10.12727/ajts.21.5 
Araújo, E. (1 de febrero de 2021). Turismo de Galicia recomienda, por error, un local de alterne de Ordes. La Voz de Galicia.

https://www.lavozdegalicia.es/noticia/santiago/2020/11/28/turismo-galiciarecomienda-error-local-alterne-ordes/0003_202011S28C7992.htm

Araújo Vila, N., Fraiz Brea, J. A. y Toubes, D. R. (2020). El turismo poscoronavirus en Galicia. Plan de reactivación. Turismo pos-COVID-19: Reflexiones, retos y oportunidades, 201-209. https://doi.org/10.25145/b.turismopos-covid-19.2020

Berengueras, J. M. (24 de agosto de 2016). El terrorismo y la geopolítica cambian el mapa de destinos turísticos. El Periódico. Recuperado el septiembre de 2016, de http://www.elperiodico.com/es/economia/20160823/turismo-consecuenciasterrorismo-cambios-destinos-5339905

Calleja-Reina, M. A., Paniagua Rojano, F. J. y Victoria Mas, J. S. (2018). Herramientas digitales y Comunicación de Crisis: El papel de las redes sociales según la voz de los expertos (2015). Estudios sobre el Mensaje Periodístico, 24(2), 717-733.

https://doi.org/10.5209/esmp.62206

Cortés, C. (9 de noviembre de 2020). El Camino de Invierno se estrena en el año jacobeo más difícil. La Voz de Galicia.

https://www.lavozdegalicia.es/noticia/lugo/2020/11/09/camino-invierno-estrena-anojacobeo-dificil/0003_202011L9C1991.htm

Dans, E. (2007). La empresa y la web 2.0. Harvard Deusto marketing \& ventas, 80, 3643. http://ipgo.webs.upv.es/mediawiki/images/7/76/La_empresa_y_la_web_2.0.pdf Domínguez Vila, T. y Araújo Vila, N. (2014). Gestión de las redes sociales turísticas en la web 2.0. Vivat Academia, O(129), 57-78. https://doi.org/10.15178/va.2014.129.57-78

E.A.E. (2 de febrero de 2021). Turismo recibe la información de Ordes para subsanar los polémicos errores de su web. La Voz de Galicia. https://www.lavozdegalicia.es/noticia/santiago/2021/02/02/turismo-recibeinformacion-ordes-subsanar-polemicos-errores-web/0003_202102S2C5992.htm 
Figueroa, E. T. y Gómez-Zorrilla, J. (2020). Marketing de crisis: Nuevas oportunidades después del COVID-19 (1.a ed.). Independently published.

Hall, C. M., Scott, D. y Gössling, S. (2020). Pandemics, transformations and tourism: be careful what you wish for. Tourism Geographies, 22(3), 577-598.

https://doi.org/10.1080/14616688.2020.1759131

IAB Spain. (2021, febrero). Top tendencias digitales 2021.

https://iabspain.es/download/49562/

Kotler, P. (2011). Marketing Turístico (5.a ed.). PRENTICE HALL/PEARSON.

Latorre, M. (2018). HISTORIA DE LAS WEB, 1.0, 2.0, 3.0 y 4.0. Universidad Marcelino Champagnat.

https://umch.edu.pe/arch/hnomarino/74_Historia\%20de\%20la\%20Web.pdf

Lugilde, A. (2020, 22 agosto). Brote en el camino de Santiago por una peregrina que no se esperó a ver su PCR. La Vanguardia.

https://www.lavanguardia.com/vida/20200822/482929383173/camino-de-santiagocovid-brote-peregrina-madriena.html

Molaviajar. (3 de septiembre de 2020). El mejor banco del mundo y Combarro. Galicia Molaviajar [Vídeo]. YouTube. https://www.youtube.com/watch?v=Juks85Vn3t4.

Neuburger, L. y Egger, R. (2020). Travel risk perception and travel behaviour during the COVID-19 pandemic 2020: a case study of the DACH region. Current Issues in Tourism, 1-14. https://doi.org/10.1080/13683500.2020.1803807

Organización de las Naciones Unidas (ONU). (2020, agosto). La COVID-19 y la transformación del turismo.

https://www.un.org/sites/un2.un.org/files/policy_brief_covid19_and_transforming_tourism_spanish.pdf 
Polo, A. (2020). Economic crisis and tourism crisis in the conditions of COVID-19. The case of Albania. In Education, Law, Business, 55-59.

http://conferencii.com/files/archive/2020-04.pdf\#page=55

Pombo, A. (12 de julio de 2017). Turismo de masas y Camino de Santiago. Gronze.com. https://www.gronze.com/articulos/turismo-masas-y-camino-santiago-13941

Prokopenko, O., Larina, Y., Chetveryk, O., Kravtsov, S., Rozhko, N. y Lorvi, I. (2019).

Digital-Toolkit for Promoting Tourist Destinations. International Journal of Innovative Technology and Exploring Engineering, 8(12), 4982-4987.

https://doi.org/10.35940/ijitee.13745.1081219

Quintero Santos, J. L. (2016). Vulnerabilidad de la Economía Española en los Marcos de la Crisis Económica. Revista Latinoamericana de Turismología, 2(1), 19-30.

https://periodicos.ufjf.br/index.php/rlaturismologia/article/view/9964

Sánchez, J. (4 de noviembre de 2020). Viajes National Geographic.

viajes.nationalgeographic.com.es.

https://viajes.nationalgeographic.com.es/lifestyle/viajes-se-trasladan-a-tiktok-nuevaventana-mundo_16125/1

Sigala, M. (2020). Tourism and COVID-19: Impacts and implications for advancing and resetting industry and research. Journal of Business Research, 117, 312-321.

https://doi.org/10.1016/j.jbusres.2020.06.015

Tsao, Cy Ni, C (2015). Vulnerability, resilience, and the adaptive cycle in a crisis-prone tourism community. Tourism Geographies, 18(1), 80-105.

https://doi.org/10.1080/14616688.2015.1116600

Turismo de Galicia. (2020, 29 diciembre). Posta en común do proceso participativo "Plan Director Turismo 21-23 Galicia Destino Seguro" [Vídeo]. YouTube. https://www.youtube.com/watch?v=-L81W06r_U0 
Vargas-Sánchez, A. (2020). Entender el turismo post-coronavirus: posibles escenarios. El turismo después de la pandemia global: análisis, perspectivas y vías de recuperación, 6-19. https://aecit.org/uploads/public/DOCUMENTO.covid-19\%20y\%20turismo.pdf Vargas-Sánchez, A. (2020a). Estrategias de destinos y empresas en el mercado turístico pos-COVID-19. Turismo pos-COVID-19. Reflexiones, retos y oportunidades, 147-158. https://doi.org/10.25145/b.Turismopos-COVID-19.2020

Xunta de Galicia. (18 de noviembre de 2020). La Xunta diseñará con el sector turístico y las tres universidades gallegas el Plan Director 2021-2023... [Comunicado de prensa]. https://www.xunta.gal/notas-de-prensa/-/nova/53201/xunta-disenara-con-sectorturistico-las-tres-universidades-gallegas-plan-director?langld=es_ES

Xunta de Galicia. (2020a). Red pública de Albergues del Camino de Santiago en Galicia. Albergues Xacobeo. https://alberguesxacobeo.gal/

Xunta de Galicia. (3 de enero de 2021). El Plan Director Galicia Destino Seguro de la Xunta recoge la necesidad de apostar por un modelo turístico... [Comunicado de prensa]. https://www.xunta.gal/notas-de-prensa/-/nova/54408/plan-director-galiciadestino-seguro-xunta-recoge-necesidad-apostar-por-modelo?langld=es_ES 